\title{
El sistema cerrado en la prevención de las infecciones del torrente sanguíneo
}

\section{The closed infusion system in the prevention of bloodstream infections}

\author{
Carlos Álvarez, Idal Beer, luis Fernando Cifuentes • \\ BOGOTÁ, D.C. (COLOMBIA)
}

\begin{abstract}
Resumen
Los pacientes hospitalizados están en continuo riesgo de infecciones del torrente sanguíneo (ITS), especialmente en las unidades de cuidado intensivo (UCI). Las ITS constituyen 14\% de las infecciones nosocomiales (IN) y representan casi un tercio de las muertes asociadas con IN. Es así que se han establecido pautas de prevención y protocolos de manejo para reducir el impacto de las ITS asociadas a catéteres (CR). Entre ellas están las diferencias entre contenedores de infusión intravenosa (IV). Los de infusión abierto necesitan la introducción de aire para su vaciamiento. Los de infusión cerrado son contenedores colapsables que no requieren aire para su vaciamiento. En conclusión, en la era de la seguridad del paciente se hace necesario que los sistemas de salud propendan por hacer los cambios en la tecnología que redundan en una mejor atención y que muchas veces son costo/efectivas (Acta Med Colomb 2011; 36: 105-107).

Palabras clave: sistema cerrado, catéteres centrales.
\end{abstract}

\begin{abstract}
Hospitalized patients are at continued risk of bloodstream infections (BSI), especially in intensive care units (ICU). BSI constitute 14\% of nosocomial infections (HAI) and represent almost a third of deaths associated with HAI. It is well established that prevention guidelines and management protocols reduce the impact of BSI associated with catheters (CR). Among them are the differences between containers intravenous (IV). The open infusion required the introduction of air for emptying. The closed infusion are collapsible containers that do not require air for emptying. In conclusion, in the era of patient safety it is necessary to propitiate health systems to make the changes in technology that result in improved care and often are cost-effective (Acta Med Colomb 2011; 36: 105-107).
\end{abstract}

Key words: closed system, central catheter.
Dr. Carlos Álvarez-Moreno: MSc, Infectólogo y Epidemiólogo Clínico, Hospital Universitario San Ignacio, Pontifica Universidad Javeriana; Dr. Idal Beer: MBA, Intensivista, Director Medical Affairs, Región Latin America Baxter Export Corporation, Ft. Lauderdale (USA); Dr. Luis Fernando Cifuentes: MSc, Farmacólogo Clínico, Director Médico, Laboratorios Baxter S.A., Bogotá, D.C. (Colombia).

Correspondencia. Dr. Luis Fernando Cifuentes.

Email: luis_cifuentes@baxter.com

Institución donde fue realizado el trabajo: ninguna.

Presentación a congresos: ninguna

Patrocinio financiero: ninguno

Recibido: 04/IV/2011 Aceptado: 14/IV/2011
Los pacientes hospitalizados están en continuo riesgo de infecciones del torrente sanguíneo (ITS), especialmente en las unidades de cuidado intensivo (UCI). Las ITS constituyen $14 \%$ de las infecciones nosocomiales (IN) y representan casi un tercio de las muertes asociadas con IN (1). La gran mayoría de ITS se relacionan con el uso de catéteres centrales (CR-ITS) y se asocian a un aumento en la hospitalización, e incremento en los costos al sistema de salud y mortalidad $(2,3)$. Las tasas de incidencia de CR-ITS para el sistema de vigilancia de los centros para la prevención y el control de enfermedades (CDC, Center for Disease Control and Prevention), la Secretaria Distrital de Salud de Bogotá y del consorcio para el control de la infección nosocomial (INNIC, International Nosocomial Infection Control Consortium) son 2.0, 2.4 y 7.0/1000 días catéter, respectivamente. Recientemente el CDC ha actualizado las guías de prevención de las CR-ITS (3) y paralelamente varias sociedades científicas colombianas adaptaron y resumieron las principales estrategias para la prevención de CR-ITS, entre las que figura nuevamente la educación y el entrenamiento de trabajadores de la salud, que sumado a la introducción de listas de chequeo, uso de antisépticos como clorhexidina, higiene de manos y el uso de sistemas cerrados como componentes claves para reducir su incidencia (4).

Las bacterias tienen dos formas para acceder a la punta del catéter: externamente, desde un entorno periférico del catéter; e internamente, desde el interior de la punta del catéter (Figura 1). La contaminación externa es la predominante, sobre todo en el momento de la inserción. Con el uso de catéteres más largos, las fuentes intraluminales se han convertido en la causa prominente de bacteriemia adquirida en el hospital (5). Durante la preparación y ad- 


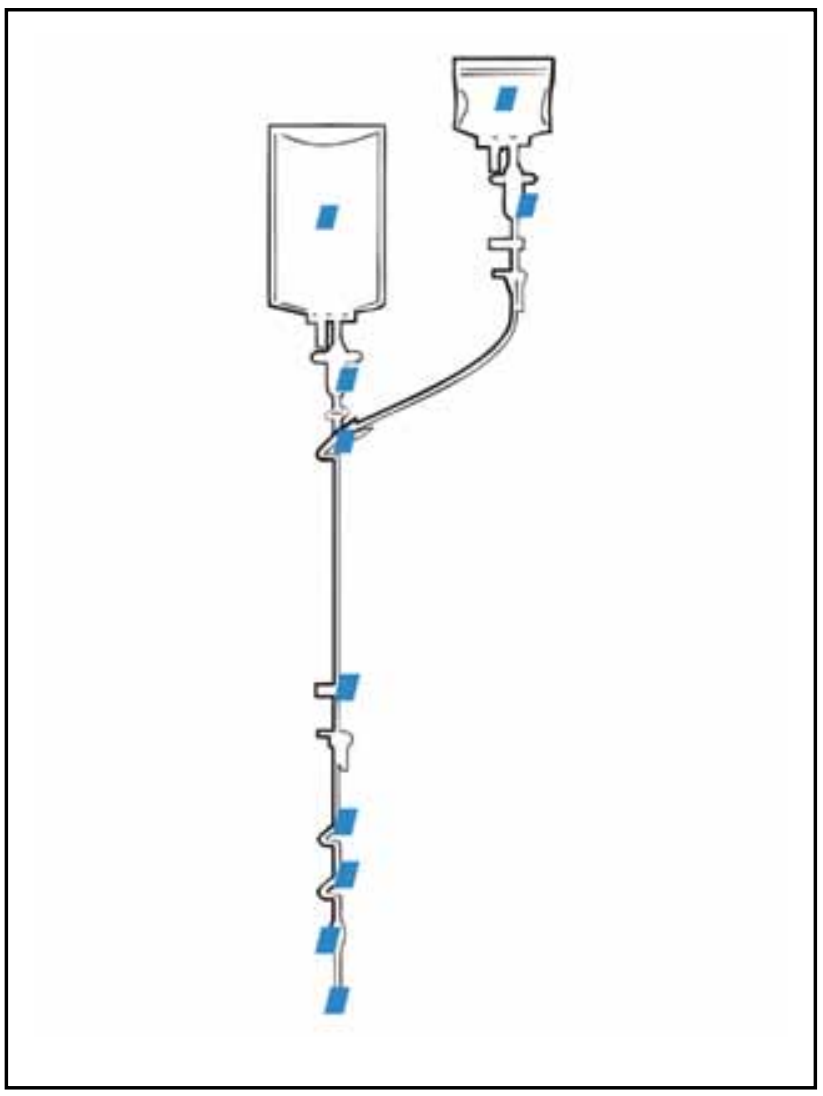

Figura 1. Puntos de contaminación potencial (marcados en azul).

ministración de líquidos endovenosos existe un alto riesgo de contaminación y adicional de contaminación extrínseca cuando el sistema se ventila o purga, lo cual es mandatorio en los sistemas de infusión abierto (2). Este riesgo es más alto en hospitales de América Latina y en países con recursos limitados (6).

Existen dos tipos de contenedores de infusión intravenosa (IV), cerrado y abierto (Figura 2) que se usan mundialmente. Los de infusión IV abierto son rígidos (vidrio) o semirrígidos (plástico) que necesitan la introducción de aire para su vaciamiento (filtro de aire o aguja). Los sistemas de infusión IV cerrado son contenedores plásticos flexibles totalmente colapsables que no requieren aire para su vaciamiento y por tanto sin volumen residual significativo; los puertos de inyección e inserción del equipo de administración son independientes y autosellantes $(2,5)$.

El uso de los sistemas cerrados flexibles de infusión IV cerrado ha venido reemplazando los sistemas abiertos en Estados Unidos y países occidentales, como estrategia estándar en la prevención de infecciones asociadas al cuidado de la salud (3). En el estudio de Rosenthal V (6), las infecciones del torrente sanguíneo se redujeron en $64 \%$ por medio del uso de sistemas de infusión IV cerrados. Un metaanálisis de cuatro cohortes (11) evaluó el impacto de cambio de contenedor abierto a cerrado, resultó en reducción

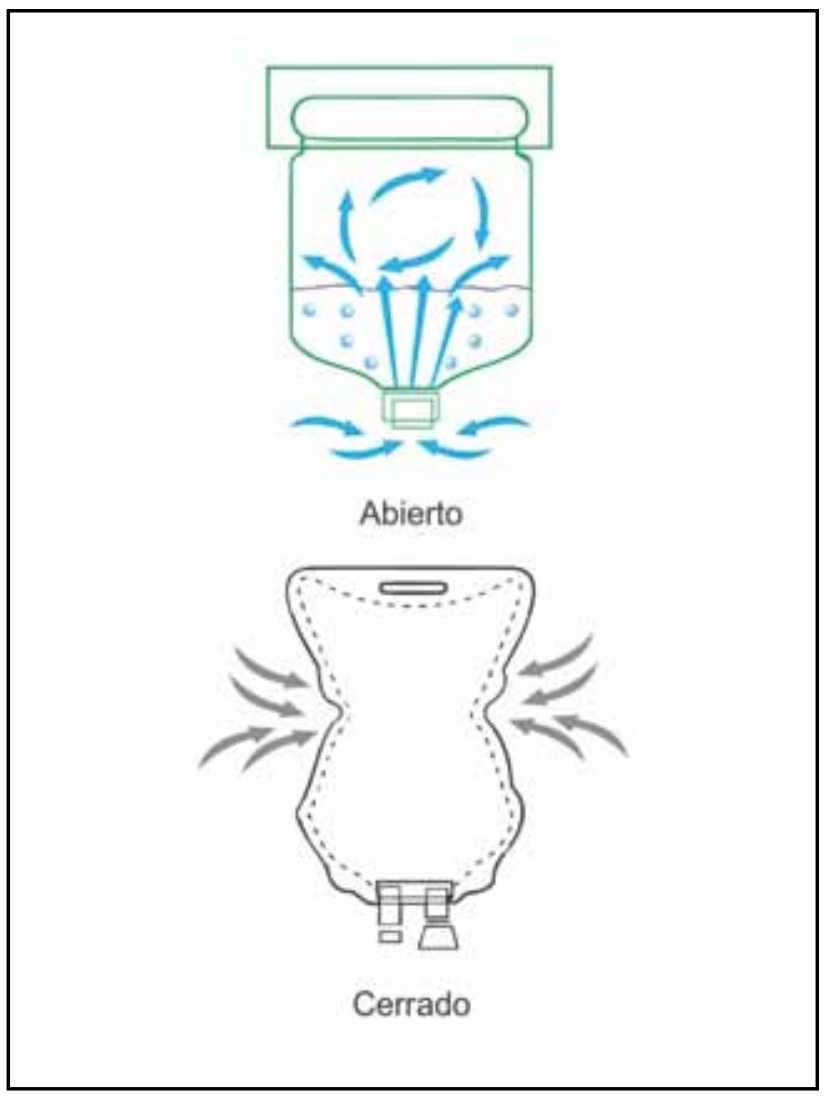

Figura 2. Sistema de infusión IV. Abierto y Cerrado.

de la incidencia de CR-ITS y causas de mortalidad en UCI (Figura 3), sugiriendo que los contenedores abiertos están asociados a un riesgo incrementado de ITS y mortalidad en UCI $(2,7-10)$.

Las soluciones y medicamentos que requieren combinación se consideran contenedores abiertos. Cada vez que a un envase estéril se le introduce una aguja hay una ruptura en el sistema con la posibilidad de contaminación.

El concepto de sistema cerrado debe incluir también los sistemas de administración de medicamentos como los contenedores cerrados que también contiene el medicamento como un premezclado/listos para uso o por lo menos sistemas listos para mezcla de fármacos. Lo anterior, con el fin de eliminar prácticamente la posibilidad de contaminación que se pueda producir con el contacto, e idealmente se deben preferir tecnologías libres de aguja (conectores y equipos de administración) que no permitan la apertura intermitente del sistema de inyección de medicamentos o retiros para muestras de sangre (5). Lyles et al. (5) mencionan en su reciente publicación que aparentemente existe un mejor entendimiento de que los sistemas cerrados requieren un conjunto de elementos o requerimientos además de los contenedores de solución IV y que llevaran en un futuro próximo a presenciar discusiones en busca de conceptos más integrales de sistema cerrado que incluirían entre otros, las 


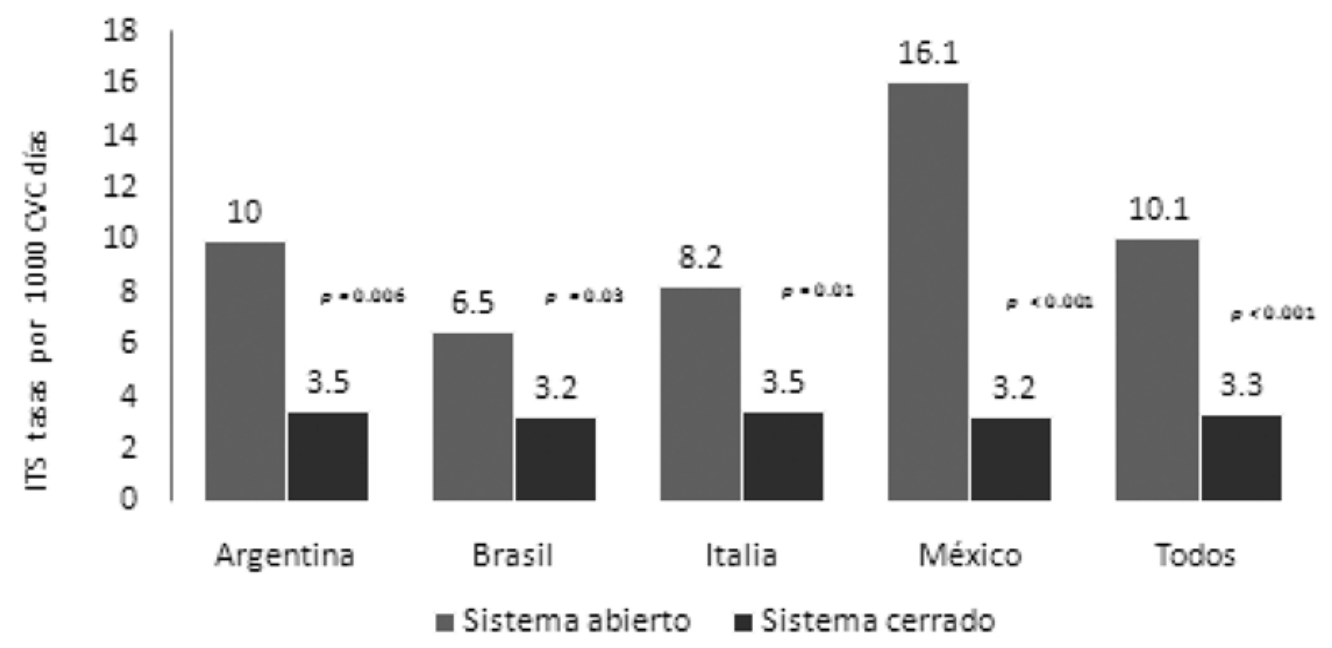

Figura 3. Resultados por país en tasas de CR-BSI x 1000 días-catéter. (Adaptado de Maki DG, et al. Infect Control Hosp Epidemiol 2011; 37: 50-8).

tecnologías sin aguja, los catéteres y equipos de administración no ventilados.

En conclusión, en la era de la seguridad del paciente se hace necesario que los sistemas de salud propendan por hacer los cambios en la tecnología que redundan en una mejor atención y que muchas veces son costo/efectivas.

\section{Referencias}

1. Kievens RM, Edwards M, Richards CL Jr, Horan TC, Gaynes RP, Pollock DA, et al. Estimating health care-associated infections and deaths in U.S. hospitals, 2002. Public Health Reports 2007; 122: 160-6.

2. Franzetti F, Borghi B, Raimondi F, Rosenthal VD. Impact on rates and time to first central vascular-associated bloodstream infection when switching from open to closed intravenous infusion containers in a hospital setting. Epidemiol Infect 2009; 137: 1041-8.

3. CDC guidelines for the prevention of CLABSI (consultado 2011 En 20. Disponible en http://www.cdc.gov/HAI/bsi/bsi.html).

4. Guías de práctica clínica para la prevención de infecciones intrahospitalarias asociadas al uso de dispositivos médicos. (consultado 2011 En. 31. Disponible en Disponible en http://acin.org/acin/new/Portals/0/Guia_IIH_Final.pdf).

5. Lyles A, Fanikos J, Whitelaw G, Bamford KB, Bouza E, Rosenthal V, et al.
Drug delivery systems' role in preventing central line-associated bacteraemia: an international perspective. EJHPPractice 2009; 15: 39-44.

6. Rosenthal VD, Maki DG, Mehta A, Alvarez-Moreno C, Leblebicioglu H, Higuera F, et al. International nosocomial infection control consortium report, data summary for 2002-2007, issued January 2008. Am J Infect Control 2008; 36: $627-37$

7. Rangel-Fausto MS, Higuera-Ramirez F, Martínez-Soto J, Rosenthal VD. Should we use closed or open infusion containers for prevention of bloodstream infections? Ann Clin Microbiol Antimicrob 2010; 9: 6.

8. Rosenthal VD, Maki DG, Salomao R, Moreno CA, Mehta Y, Higuera F, et al Device-associated nosocomial infections in 55 intensive care units of 8 developing countries. Ann Intern Med 2006; 145: 582-91.

9. Maki DG, Rosenthal VD, Salomao R, Franzetti F, Rangel-Frausto MS.. Cost effectiveness of switching from an open IV infusion system on rates of central venous catheter-associated bloodstream infection in three Mexican hospitals 2005 APIC.

10. Vilins M., Blecher S, Silva MA, Rosenthal VD, Barker K, Salomao R. Rate and time to develop first central line-associated bloodstream infections when comparing open and closed infusion containers in a Brazilian Hospital. Braz J Infect Dis 2009; 13: 335-40.

11. Maki DG, Rosenthal VD, Salomao R, Franzetti F, Rangel-Fausto MS. Impact of switching from an open to a closed infusion system on rates of central lineassociated bloodstream infection: A meta-analysis of time sequence cohort studies in 4 countries. Infect Control Hosp Epidemiol 2011; 32: 50-8. 